\title{
Enumerating tilings of rectangles by squares with recurrence relations*
}

\author{
DARYL DEFORD ${ }^{\dagger}$
}

\begin{abstract}
Counting the number of ways to tile an $m \times n$ rectangle with squares of various sizes is a traditional combinatorial problem. In this paper, we demonstrate a simple variation of the transfer matrix method for constructing the recurrence relations satisfied by the solutions to these problems. This method also generalizes to similar problems that have not been previously considered, including three-dimensional "tilings." We are able to give an upper bound on the minimal order of the recurrence satisfied for fixed $m$, as well as to prove that there does not exist a graph whose matchings form a one-to-one correspondence with such tilings.
\end{abstract}

AMS 2000 subjeCt Classifications: Primary 05B45; Secondary 52C20. KEYWORDS AND PHRASES: Recurrence relation, tilings, line graphs.

\section{Introduction}

Counting the number of rectangular board tilings with tiles of different sizes and shapes can lead to many interesting combinatorial proofs and identities. Examples of these types of problems can be found in $[1,8,9,15,19]$. In this paper we will be considering variations on the following problem:

Given an $m \times n$ rectangular board and an unlimited number of square tiles of various, fixed dimensions, in how many different ways can the board be tiled?

This problem was considered by Heubach, in 1999 [12], motivated by previous work on tiling rectangles with Cuisinaire rods by Brigham et al. [2] and Hare [11]. Heubach's approach was to construct recurrence relations for the sequences formed by fixing the row dimension of the board and letting the column dimension vary while the set of square tiles remains unchanged. The coefficients of the terms in the recurrence relations were found by count-

\footnotetext{
${ }^{*}$ This work was supported by a grant from the Washington State University College of Sciences.

${ }^{\dagger}$ I would like to express my gratitude to Dr. William Webb for his support and guidance.
} 
ing the number of "basic" blocks, those that cannot be decomposed along a vertical line, that could be formed from the given square tiles.

Unfortunately, this method grows significantly more complex as the fixed number of rows grows. Thus, it is not a method that can be widely applied, outside of small cases. In 2006, Calkin et al. showed that when the square tiles are restricted to dimensions of $1 \times 1$ and $2 \times 2$, the number of tilings can be calculated as the sum of the entries in the $n^{\text {th }}$ power of a recursively defined matrix [3]. This solution is based on a method of Calkin and Wilf and the equivalence between this problem with $1 \times 1$ and $2 \times 2$ squares and placing non-attacking kings on an $(m-1) \times(n-1)$ chessboard [4]. However, because of this equivalence, this method cannot be used to calculate the number of tilings when the square tiles are permitted to be larger than $2 \times 2$.

In this paper, we will present a method, related to the transfer matrix method, that can be used to generate recurrence relations for a much wider class of problems than has been previously considered. For example, our method applies equally well to the three-dimensional analogue of this problem, tiling $m \times n \times k$ rectangular prisms with cubes of fixed dimensions. We will also give upper bounds on the order of the recurrence satisfied by problems with fixed row dimension and square sizes. Finally, we will show that even for the simple case of $1 \times 1$ and $2 \times 2$ squares the method of matrix permanents or pfaffians cannot be used to construct a general solution. These matrix methods have been used successfully on other similar problems $[13,14,15]$.

\section{Counting rectangle tilings}

When the fixed number of rows, $m$, is small, the recurrence relations are frequently simple to generate. It is easy to see that the number of ways to tile a $2 \times n$ rectangle with $1 \times 1$ and $2 \times 2$ squares is equal to the $n^{\text {th }}$ combinatorial Fibonacci number, by comparison to $1 \times n$ tilings with squares and dominoes. A similar argument applied to the $3 \times n$ case, with squares of sizes up to $3 \times 3$, gives us the recurrence $a_{n}=a_{n-1}+2 a_{n-2}+a_{n-3}$ by simply considering what must happen to a tiling whose initial column contains a particular tile [12]. As the fixed number of rows grows, however, this problem becomes increasingly complex, and ad hoc methods cannot be counted on to provide convenient solutions.

As mentioned in the introduction, previously considered methods have been difficult to apply to either large problem instances [12] or more general sets of tiles [3]. Our method in these larger cases proceeds as follows: given 
a fixed row dimension, $m$, and set of permissible square tiles, we construct a system of linear recurrence relations that represents all of the ways to tile the initial column. A result of Webb, Criddle, and DeTemple guarantees that such a system of recurrence relations exists [20].

Once we have obtained this system of recurrences, we use the successor operator method of DeTemple and Webb [8] to express the system as a matrix whose entries are polynomials in the successor operator. Their proof (Theorem 7.20 in [8]) shows that the determinant of this matrix is an annihilating polynomial for the desired sequence and hence provides the recurrence relation satisfied by the tilings. This method is equivalent to the transfer matrix method, with the successor operator used to generate the matrix instead of a digraph as in Section 4.7 of [19]. The successor method has a wide variety of applications. A similar approach is used in [7] to enumerate rearrangements on graphs, while in [18] Panario et al. use the succcessor operator to solve conditional recurrences, and in [8] an entire chapter is devoted to applications of the method to prove recurrences and identities.

\subsection{Examples}

To demonstrate the efficacy of this method we present some examples of problems solved using our method.

Example 1. Tiling $7 \times n$ rectangles with squares of size $1 \times 1$ and $2 \times 2$.

This problem is one that can be solved by the method of Calkin et al. as the sum of the entries in the $(n-2)$ power of a $64 \times 64$ matrix. Figure 1 shows the eight endings that we must consider. Note that each of the endings shown represents an equivalence class of endings. For example, consider an ending where a single $2 \times 2$ square is placed in either the top or bottom row and the remaining squares are filled with $1 \times 1$ squares. The number of ways to cover the remaining portion of the rectangle is the same in either case, thus, we need only consider one representative of this class of endings, in this case $b_{n}$.

In order to demonstrate how the system of recurrence relations is generated, we give explicit derivations of two of the equations in our system. All of the other equations are derived in a similar fashion. The initial column of a board that ends like $c_{n}$ can be covered in five different ways (noticing that the bottommost square must be covered with a $1 \times 1$ ):

1. All of the squares may be covered with $1 \times 1$ squares leaving a board of $a_{n-1}$. 


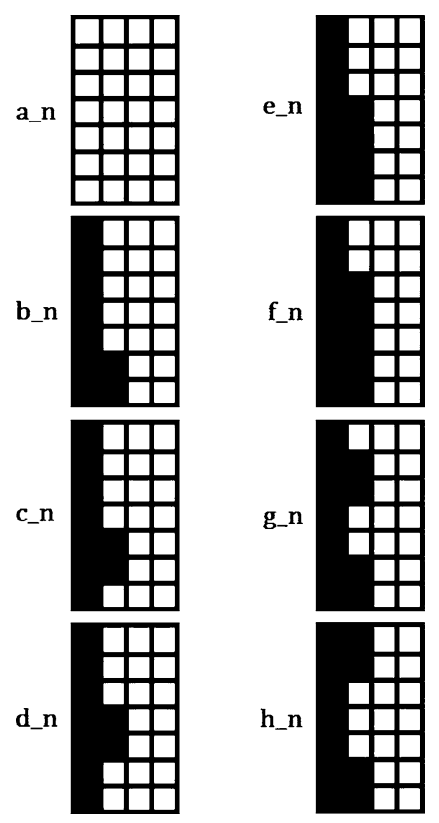

Figure 1: Endings of a $7 \times n$ Rectangle.

2. A single $2 \times 2$ square may be placed in the top row, while the rest of the column is filled with $1 \times 1$. The number of ways to tile the remaining board is $b_{n-1}$.

3. A single $2 \times 2$ square may be placed beginning in the second row, while the rest of the column is filled with $1 \times 1$. The number of ways to tile the remaining board is $c_{n-1}$.

4. A single $2 \times 2$ square may be placed beginning in the third row, while the rest of the column is filled with $1 \times 1$. The number of ways to tile the remaining board is $d_{n-1}$.

5. Finally, two $2 \times 2$ squares may be placed in the tiling, leaving a board equivalent to $e_{n-1}$.

Thus, the total number of tilings of the board $c_{n}$ is equal to $a_{n-1}+b_{n-1}+$ $c_{n-1}+d_{n-1}+e_{n-1}$. A similar method gives the equation for $g_{n}$ although there are only two possibilities in this case.

1. Both squares in the initial column may be covered with $1 \times 1$ squares leaving a board of shape $a_{n-1}$.

2. A single $2 \times 2$ square may be placed in the initial column. The number of tilings of the remaining board is then $d_{n-1}$. 
Hence, we have that $g_{n}=a_{n-1}+d_{n-1}$. Applying a similar process to all of the possible endings gives the system of recurrences shown below:

$$
\begin{array}{lc}
a_{n}= & a_{n-1}+5 a_{n-2}+2 b_{n-1}+2 c_{n-1}+2 d_{n-1}+2 e_{n-1}+4 f_{n-1}+2 g_{n-1}+h_{n-1} \\
b_{n}= & a_{n-1}+b_{n-1}+c_{n-1}+2 d_{n-1}+e_{n-1}+2 f_{n-1} \\
c_{n}= & a_{n-1}+b_{n-1}+c_{n-1}+d_{n-1}+e_{n-1} \\
d_{n}= & a_{n-1}+2 b_{n-1}+c_{n-1}+g_{n-1}+h_{n-1} \\
e_{n}= & a_{n-1}+b_{n-1}+c_{n-1} \\
f_{n}= & a_{n-1}+b_{n-1} \\
g_{n}= & a_{n-1}+d_{n-1} \\
h_{n}= & a_{n-1}+2 d_{n-1}
\end{array}
$$

Applying the successor operator to this system gives the following matrix $M$, that satisfies the equation $M \vec{x}=\overrightarrow{0}$, where $\vec{x}$ is the column vector $\left[a_{n}, b_{n}, \ldots, h_{n}\right]$ and $\overrightarrow{0}$ is the $8 \times 1$ zeros vector.

$$
M=\left[\begin{array}{cccccccc}
E^{2}-E-5 & -2 E & -2 E & -2 E & -2 E & -4 E & -2 E & -E \\
-1 & E-1 & -1 & -2 & -1 & -2 & 0 & 0 \\
-1 & -1 & E-1 & -1 & -1 & 0 & 0 & 0 \\
-1 & -2 & -1 & E & 0 & 0 & -1 & -1 \\
-1 & -1 & -1 & 0 & E & 0 & 0 & 0 \\
-1 & -1 & 0 & 0 & 0 & E & 0 & 0 \\
-1 & 0 & 0 & -1 & 0 & 0 & E & 0 \\
-1 & 0 & 0 & -2 & 0 & 0 & 0 & E
\end{array}\right]
$$

The determinant of this matrix is a characteristic polynomial of the recurrence relation that we are seeking. Thus,

$\operatorname{det}(M)=E^{9}-3 E^{8}-30 E^{7}+17 E^{6}+138 E^{5}-85 E^{4}-116 E^{3}+42 E^{2}+32 E$ gives us the recurrence satisfied by our sequence:

$a_{n}=3 a_{n-1}+30 a_{n-2}-17 a_{n-3}-138 a_{n-4}+85 a_{n-5}+116 a_{n-6}-42 a_{n-7}-32 a_{n-8}$.

Example 2. Tiling $5 \times n$ rectangles with squares of size $1 \times 1,2 \times 2,3 \times 3$, $4 \times 4$, and $5 \times 5$.

This example can be found in the original paper of Heubach where a recurrent expression is given, consisting of a convolution of two separate linear homogeneous recurrence relations with constant coefficients (dependent 


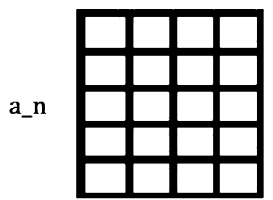

c_n

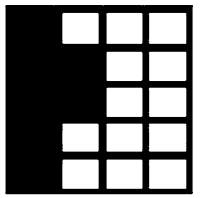

b_n

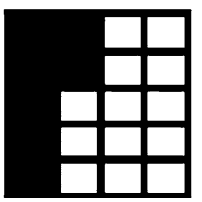

d_n

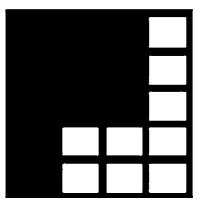

Figure 2: Endings of a $5 \times n$ Rectangle.

on $n$ ). The OEIS however, does contain an equivalent constant coefficient recurrence which we derive in this example [17]. As in the previous example, we progress by constructing a system of linear recurrences that accounts for all of the endings shown in Figure 2. Note that this problem requires fewer endings than the previous example, even though we are using a wider variety of tiles. As an intuitive explanation, for example, there is no difference in the number of tilings remaining, between a tiling that ends with a $3 \times 3$ square above a $2 \times 2$ square and a tiling with one fewer column that ends with a single $2 \times 2$ square beginning in the second row and three $1 \times 1$ squares. Both of these endings, even though they are constructed with different tiles, are counted by $c_{n}$.

Thus, applying the methods of the previous example gives us the following system of recurrences and related successor matrix:

$$
\begin{aligned}
& a_{n}=a_{n-1}+3 a_{n-2}+a_{n-3}+2 a_{n-4}+a_{n-5} \\
& +2 b_{n-1}+2 c_{n-1}+2_{n-2}+2 d_{n-1} \\
& b_{n}=a_{n-1}+b_{n-1}+c_{n-1}+d_{n-1} \\
& c_{n}=\quad a_{n-1}+b_{n-1} \\
& d_{n}=\quad a_{n-2}+c_{n-1} \\
& {\left[\begin{array}{cccc}
E^{5}-E^{4}-3 E^{3}-E^{2}-2 E-1 & -2 E^{4} & -2 E^{4}-2 E^{3} & -2 E^{4} \\
-1 & E-1 & -1 & -1 \\
-1 & -1 & E & 0 \\
-1 & 0 & -E & E^{2}
\end{array}\right]}
\end{aligned}
$$

The determinant of this matrix gives the characteristic polynomial of our desired recurrence relation, which is again an eighth order recurrence:

$$
a_{n}=2 a_{n-1}+7 a_{n-2}+6 a_{n-3}-a_{n-4}-6 a_{n-5}-a_{n-7}-2 a_{n-8} .
$$




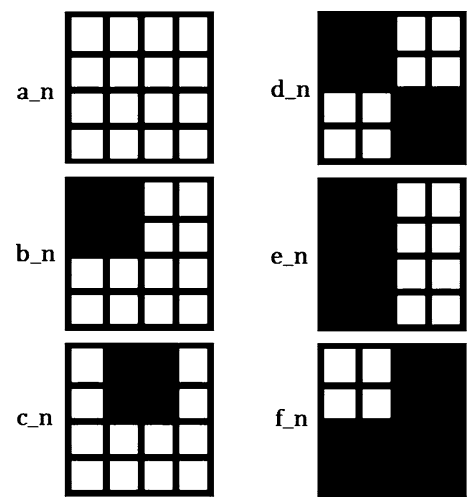

Figure 3: Endings of a $4 \times 4 \times n$ Prism.

Example 3. Tiling $4 \times 4 \times n$ rectangular prisms with cubes of size $1 \times 1 \times 1$, $2 \times 2 \times 2,3 \times 3 \times 3$, and $4 \times 4 \times 4$.

Our final example shows how this method can be extended to other types of related tilings problems, including those in three dimensions. Figure 3 shows the "endings" that we must consider in this example. Note that the initial column we are covering in order to construct our recurrences is a $4 \times 4 \times 1$ region, so the tilings in the figure show only the first dimensional placements of $2 \times 2 \times 2$ cubes, with the assumption that $1 \times 1 \times 1$ cubes fill the remaining squares. Obviously, a $4 \times 4 \times 4$ cube fills all of the spaces available, and a $3 \times 3 \times 3$ cube must be surrounded by $211 \times 1 \times 1$ cubes filling in the gaps.

Again, the endings considered here represent equivalence classes of endings. To further illustrate this point, note that $c_{n}$ and $e_{n}$ actually count the same number of tilings, since the single $2 \times 2 \times 2$ cube in $c_{n}$ must be surrounded by eight $1 \times 1 \times 1$ cubes. Similarly, if a single $2 \times 2 \times 2$ cube is placed in the center location of the last column, it must be surrounded by $241 \times 1 \times 1$ cubes and the remaining prism can be tiled in $a_{n-2}$ ways.

Based on the endings shown in Figure 3, we obtain the following system of recurrences and successor matrix:

$$
\begin{aligned}
& a_{n}=\quad a_{n-1}+8 a_{n-2}+4 a_{n-3}+a_{n-4} \\
& +4 b_{n-1}+4 c_{n-1}+2 d_{n-1}+4 e_{n-1}+12 f_{n-1} \\
& b_{n}=a_{n-1}+3 b_{n-1}+2 c_{n-1}+d_{n-1}+2 e_{n-1}+3 f_{n-1} \\
& c_{n}=\quad a_{n-1}+2 b_{n-1}+c_{n-1}+e_{n-1} \\
& d_{n}=\quad a_{n-1}+2 b_{n-1}+d_{n-1}
\end{aligned}
$$




$$
\begin{aligned}
& e_{n}=\quad a_{n-1}+2 b_{n-1}+c_{n-1}+e_{n-1} \\
& f_{n}=\quad a_{n-1}+b_{n-1} \\
& {\left[\begin{array}{cccccc}
E^{4}-E^{3}-8 E^{2}-4 E-1 & -4 E^{3} & -4 E^{3} & -2 E^{3}-4 E^{3} & -12 E^{3} & \\
-1 & E-3 & -2 & -1 & -2 & -3 \\
-1 & -2 & E-1 & 0 & -1 & 0 \\
-1 & -2 & 0 & E-1 & 0 & 0 \\
-1 & -2 & -1 & 0 & E-1 & 0 \\
-1 & -1 & 0 & 0 & 0 & E
\end{array}\right]}
\end{aligned}
$$

This gives us a recurrence for the number of ways to tile a $4 \times 4 \times n$ rectangular prism with cubes:

$a_{n}=7 a_{n-1}+28 a_{n-2}-123 a_{n-3}+18 a_{n-4}+84 a_{n-5}+20 a_{n-6}+a_{n-7}-2 a_{n-8}$.

\section{Recurrence order bounds}

In the previous section we showed how to construct a recurrence relation for a given set of square tiles and fixed rectangle row dimensions. The three examples we presented had different dimensions and different sets of permissible tiles, but in each case the order of the recurrence relation that we obtained was eight. In this section we give an explicit upper bound on the order of recurrence for a tiling problem with $1 \times 1$ and $2 \times 2$ squares that depends on the fixed number of rows and demonstrate how this procedure could be applied to more general cases.

Proposition 1. For any fixed natural number $m$, the minimal order recurrence relation counting the number of ways to tile a $m \times n$ board with $1 \times 1$ and $2 \times 2$ squares has degree at most $f_{m}$.

Proof. Throughout this section we will assume that $a_{n}$ represents the number of legitimate tilings of a complete board as in the examples. As noted in Section 2.1, the number of ways to tile a $2 \times n$ strip with $1 \times 1$ and $2 \times 2$ squares is equal to $f_{n}$. This implies that there are $f_{m}$ ways to cover the initial column with these tiles. Since the maximum length of any tile is 2 , the system of recurrences can be constructed so that every recurrence except $a_{n}$ is written as a sum of other recurrence elements with minimum index $n-1$, while the sum equal to $a_{n}$ will contain an $a_{n-2}$ term.

Since there are at most $f_{m}$ endings subject to the previous conditions, the successor matrix can have at most $f_{m}$ rows, each of whose highest power of the successor operator is 1 except the row representing $a_{n}$ which may contain $E^{2}$. Since the tiling with all $1 \times 1$ squares leaves $a_{n-1}$ tilings, this gives us an upper bound on the order of the recurrence as $f_{m}+1-1=f_{m}$. 
Table 1: Comparison between the derived bound and the actual order

\begin{tabular}{|l|c|c|c|c|c|c|c|c|c|c|}
\hline Number of rows $(m)$ & 1 & 2 & 3 & 4 & 5 & 6 & 7 & 8 & 9 & 10 \\
\hline Observed Recurrence Order $[17]$ & 1 & 2 & 2 & 3 & 4 & 6 & 8 & 14 & 19 & 32 \\
\hline Upper Bound $\left(f_{m}\right)$ & 1 & 2 & 3 & 5 & 8 & 13 & 21 & 34 & 55 & 89 \\
\hline
\end{tabular}

Table 1 shows the differences between this bound and the actual order of the computed recurrence for the first several cases. The values in the center row are the orders of recurrences given in the OEIS for the solutions of these problems for each fixed $m$ [17].

Certainly, Table 1 shows that the bound given in Proposition 1 is not tight. As noted in Example 1, each ending that needs to be considered for the recurrence system represents a class of symmetric endings, each of which has the same number of rearrangements. Thus, a better bound can be obtained by considering only the distinct endings up to symmetry since these correspond more directly to rows in the successor matrix. Using these ideas, a better bound is given in [6], where these ideas are developed in more generality. However, since the process of passing to the classes of endings only reduces the total number of sequences in the system by a finite factor for each considered ending it does not change the asymptotic value of the bound which is still governed by the largest eigenvalue of the bounding sequence, in this case $\varphi=\frac{1+\sqrt{5}}{2}$.

Computing the order of recurrences for other sets of tiles can be done in an equivalent fashion. In order to obtain a similar bound for an arbitrary problem, we need only to compute the possible number of endings, since they correspond to rows in the successor matrix, and multiply this value by the side length of the largest square since this is a bound on the degree of each entry in the matrix. Additionally, it is simple to bound the number of possible endings since this amounts to solving a $1 \times n$ tiling problem, discussed extensively in [1]. Further analysis using the symmetry classes of the tiling endings can lead to more complex upper bounds, which can be stated in terms of recurrent sequences [6].

\section{Matchings and tilings}

In general, it is very difficult to provide a closed-form expression that counts the number of tilings of some rectangle, when we allow both the number of rows and columns to be arbitrarily chosen. One method that can be used to construct such an expression requires constructing a family of graphs whose matchings have a one-to-one correspondence with the tilings. Then, matrix 
permanents or pfaffians can sometimes be used to generate such a closed form. Also, for some similar problems matrix permanents can be used to generate the initial conditions that are necessary to make full use of the derived recurrence relations.

For example, Kasteleyen's formula for the number of domino tilings of an $m \times n$ rectangle uses pfaffians to count the perfect matchings in a grid graph $[10,15]$. Similarly, Kuperberg's survey gives an overview of many combinatorial problems that can be solved with the permanent-determinant method [14], while Lundow demonstrates the permanent method for matchings on hypercubes [16], which can be considered $n$-dimensional cube tilings. Here, we prove that this method will not work for enumerating tilings of an $m \times n$ rectangle with $1 \times 1$ and $2 \times 2$ squares.

Theorem 1. There does not exist a simple graph whose matchings are in a one-to-one correspondence with the number of tilings of an $m \times n$ rectangle with $1 \times 1$ and $2 \times 2$ squares, when $m \geq 4$ and $n \geq 4$.

Proof. Let $m$ and $n$ be any two integers greater than 3 and consider an $m \times n$ rectangle, $R_{m, n}$. Assume that there exists a graph, $H_{m, n}$, such that there exists a structure-preserving bijection between matchings on $H_{m, n}$ and legitimate tilings on $R_{m, n}$. We will argue by contradiction.

Construct another graph, $G_{m, n}$, such that the vertices of $G_{m, n}$ represent the $(m-1)(n-1)$ possible positions that a $2 \times 2$ square could be placed on $R_{m, n}$. Add an edge to $G_{m, n}$ between any two vertices if and only if a $2 \times 2$ square placed at the position represented by the first vertex would overlap a $2 \times 2$ square placed at the position represented by the second vertex.

Observe that the graph shown in Figure 4 is isomorphic to $G_{4,4}$. Furthermore, we see that for any $m, n \geq 4$, it must be that $G_{m, n}$ has at least one vertex-induced sub-graph isomorphic to $G_{4,4}$. We now claim that there exists a one-to-one correspondence between tilings of $R_{m, n}$ with $1 \times 1$ and $2 \times 2$ squares and independent vertex sets on $G_{m, n}$.

In order to demonstrate a bijection between these two objects consider an arbitrary tiling of an $m \times n$ rectangle. To construct a unique independent vertex set from this tiling, select each vertex whose corresponding $2 \times 2$ square appears in the tiling. These vertices must be independent since a legitimate tiling cannot contain overlapping $2 \times 2$ squares.

To construct a unique tiling from a given, arbitrary independent set place a $2 \times 2$ square in the tiling for each vertex in the set and fill the rest of the squares with $1 \times 1$ tiles. Thus, we have a structure-preserving bijection between independent vertex sets on $G_{m, n}$ and tilings of $R_{m, n}$. This correspondence demonstrates that these two objects count the same sequence. 


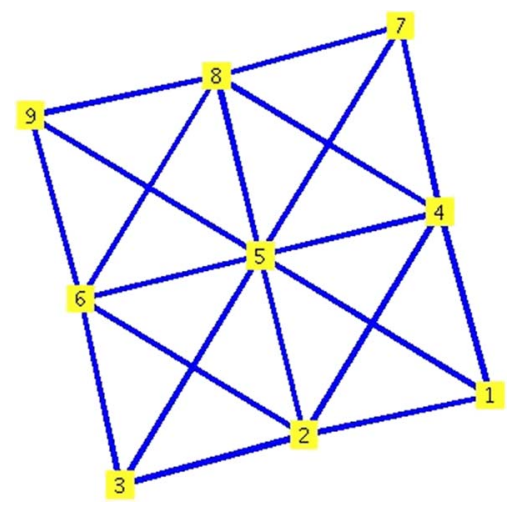

Figure 4: The Independent Set Graph $G_{4,4}$.

For any graph there is a one-to-one correspondence between matchings on the graph and independent sets on its line graph. Also, with the exception of $K_{1,3}$ and $K_{3}$ a graph is uniquely recoverable from its line graph. Since by assumption the matchings of $H_{m, n}$ correspond to tilings on $R_{m, n}$, it must be the case that the line graph of $H_{m, n}$ must be $G_{m, n}$.

However, by Beineke's forbidden minor theorem for line graphs [5], $G_{4,4}$, and hence any graph that contains $G_{4,4}$ as an induced sub-graph, cannot be the line graph of any simple graph. Thus, we have shown that $G_{m, n}$ cannot be the line graph of any simple graph. This contradicts the existence of $H_{m, n}$ and our proof is complete.

Theorem 2. There does not exist a simple graph whose maximal matchings are in a one-to-one correspondence with the number of tilings of an $m \times n$ rectangle with $1 \times 1$ and $2 \times 2$ squares, when $m \geq 4$ and $n \geq 4$.

Proof. Let $m$ and $n$ be any two integers greater than 3 and consider an $m \times n$ rectangle, $R_{m, n}$. Assume that there exists a graph, $H_{m, n}$, such that there exists a structure-preserving bijection between maximal matchings on $H_{m, n}$ and legitimate tilings on $R_{m, n}$. We will argue by contradiction.

As in the previous proof, construct a graph $F$ whose $[m n+(m-1)(n-1)]$ vertices represent the possible positions of squares in a tiling of $R_{m, n}$, and again join two edges if and only if the squares they represent would overlap if both placed in a single tiling of $R_{m, n}$. It is easy to see that this graph contains $G_{m, n}$ as an induced sub-graph.

Now notice that maximal independent sets in $F_{m, n}$ correspond in a oneto-one fashion with tilings of $R_{m, n}$ via a bijection similar to the one presented in the proof of Theorem 1 . In this case the $1 \times 1$ tiles are selected 
directly into a tiling by being members of a maximum independent set, since a vertex representing a $1 \times 1$ tile in $F_{m, n}$ is only adjacent to vertices that represent $2 \times 2$ tiles. Similarly, when constructing a unique independent set from a given tiling the $1 \times 1$ vertices have to be added to the set explicitly, but the procedure is still one-to-one.

Again, by the argument given above, since $F_{m, n}$ contains $G_{4,4}$ as an induced sub-graph it cannot be the line graph of any simple graph. This contradicts our assumption that $H_{m, n}$ exists and completes the proof.

\section{References}

[1] A. Benjamin and J. Quinn (2003). Proofs that Really Count. MAA, Washington D.C. MR1997773

[2] R. Brigham, R. Caron, P Chinn, and R. Grimaldi (1996). A Tilings Scheme for the Fibonacci Numbers. Journal of Recreational Mathematics, 28 (1), 10-17.

[3] N. Calkin, K. James, S. Purvis, S. Race, K. Schneider, and M. Yancey (2006). Counting Kings: Explicit Formulas, Recurrence Relations, and Generating Functions! Oh My! Congressus Numerantium, 182, 41-51. MR2311287

[4] N. Calkin and H. Wilf (1998). The Number of Independent Sets in a Grid Graph. SIAM J. Discrete Math, 11, 54-60. MR1612853

[5] G. Chartrand, L. Lesniak, and P. Zhang (2011). Graphs \& Digraphs 5th ed. CRC Press, Boca Raton. MR2766107

[6] D. DeFord. Enumerating Distinct Chessboard Tilings. Submitted to Fibonacci Quarterly.

[7] D. DeFord (2014). Seating Rearrangements on Arbitrary Graphs. Involve, 7 (6), 787-805. MR3284885

[8] D. DeTemple and W. Webb (2014). Combinatorial Reasoning: An Introduction to the Art of Counting. Wiley, Hoboken. MR3237721

[9] R. Graham, D. Knuth, and O. Patashnik (1994). Concrete Mathematics, 2nd ed. Addison-Wesley, Reading. MR1397498

[10] F. Harary (1967). Graph Theory and Theoretical Physics. Academic Press, New York. MR0230648

[11] E. Hare (1994). Tiling a $3 \times n$ Area with Cuisinaire Rods of Length Less Than or Equal to $k$. Congressus Numerantium, 105, 34-55. MR1382434 
[12] S. Heubach (1999). Tiling an $m$-by $-n$ Area with Squares of Size up to $k-$ by $-k(m \leq 5)$. Congrssus Numerantium, 140, 43-64. MR1745208

[13] P. Kasteleyn (1961). The Statistics of Dimers on a Lattice: I. The Number of Dimer Arrangements on a Quadratic Lattice. Physica, 27 (12), $1209-1225$.

[14] G. Kuperberg (1998). An Exploration of the Permanent-Determinant Method. Electronic Journal of Combinatorics, 5, R46: 1-34. MR1663576

[15] N. Loehr (2011). Bijective Combinatorics. CRC Press, Boca Raton. MR2777360

[16] P. Lundow (1996). Computation of matching polynomials and the number of 1-factors in polygraphs. Research Reports Umeå, 12.

[17] OEIS Foundation Inc. (2014). The On-Line Encyclopedia of Integer Sequences. http://oeis.org.

[18] D. Panario, S Murat, Q. Wang, and W. Webb (2014). General Conditional Recurrences. Applied Mathematics and Computation, 243, 220231. MR3244472

[19] R. Stanley (2012). Enumerative Combinatorics, Volume 1, 2nd ed. Cambridge University Press, New York. MR2868112

[20] W. Webb, N. Criddle, and D. DeTemple (2009). Combinatorial Chessboard Tilings. Congressus Numereratium, 194, 257-262. MR2463544

DARYl DeFord

Department of Mathematics

Dartmouth College

E-mail address: ddeford@math.dartmouth.edu

URL: http://www.math. dartmouth.edu/ ddeford

Received 5 OCtober 2012 